\title{
EFECTO DE LA AVENA Y/O LOVASTATINA SOBRE EL PERFIL LIPÍDICO EN PACIENTES DISLIPIDÉMICOS DEL HOSPITAL DE TUNJUELITO, BOGOTÁ 2008-2009
}

\section{EFFECT OF OATS AND/OR LOVASTATIN ON LIPID PROFILE IN DYSLIPIDEMIC PATIENTS OF THE TUNJUELITO HOSPITAL, BOGOTA 2008-2009}

\author{
Aura Mónica Alarcón Galeano ${ }^{1}$, Diego Felipe Buitrago Hernández ${ }^{2}$, Laura Angélica Romero Fierro ${ }^{3}$, Ana María Sánchez
} Leyva ${ }^{4}$, William Onatra H. ${ }^{5}$, María Teresa Ríos ${ }^{6}$

\begin{abstract}
${ }^{1}$ Médico Cirujano, Facultad de Medicina, Universidad de Ciencias Aplicadas y Ambientales U.D.C.A. Bogotá, Colombia. Residente de Oftalmología Hospital de La Habana, Cuba; ${ }^{2}$ MD. U.D.C.A; ${ }^{3}$ MD. U.D.C.A, ange_344@ hotmail.com; ${ }^{4}$ MD. U.D.C.A. Médica. Colsanitas, pitituta@hotmail.com; ${ }^{5}$ Profesor Titular, Facultad de Medicina, Universidad de Ciencias Aplicadas y Ambientales U.D.C.A, wonatra@gmail.com; ${ }^{5}$ Estadística, Docente Universidad de Ciencias Aplicadas y Ambientales U.D.C.A, Calle 222 No. 55-30, Bogotá, Colombia. materito@gmail.com.
\end{abstract}

Rev. U.D.C.A Act. \& Div. Cient. 16(2): 319-326, Julio-Diciembre, 2013

\section{RESUMEN}

Se ha postulado que dietas ricas en fibras son útiles para disminuir el riesgo cardiovascular. Entre los medicamentos hipolipemiantes más usados, se encuentran las estatinas, los fibratos y, en 1997, la FDA aprobó la avena. La presente investigación tuvo como objetivo determinar el efecto de avena y de lovastatina en el perfil lipídico, de pacientes dislipidémicos. La muestra de pacientes estuvo constituida por un total de 84 con dislipidemia, del hospital de Tunjuelito, en Bogotá. Se distribuyeron al azar y la administración de los medicamentos, se realizó en forma aleatorizada. Al Grupo 1, se le administró Avena (A), 29 casos (34,5\%). Grupo 2, Avena más Lovastatina $(A+L), 22$ casos $(26,2 \%)$ y, el Grupo 3, Lovastatina (L), 33 casos (39,3\%). Se hizo seguimiento durante doce semanas. Los datos, se procesaron en el programa estadístico SPSS. El IMC no mostró diferencias en el grupo 1, siendo más significativa en el grupo 2 que el en grupo 3 . El perímetro abdominal evidencia diferencias significativas en los tres grupos. El colesterol mostró diferencias significativas entre el grupo 1 y 3 . Los triglicéridos manifestaron significancia en los grupos 2 y 3 . El presente estudio evidencia cómo los pacientes dislipidémicos se benefician al agregar un producto natural, como la avena, en los parámetros de perímetro abdominal y colesterol.

Palabras clave: Hipolipemiante, fibratos, perímetro abdominal, colesterol.

\section{SUMMARY}

It has been postulated that diets rich in fiber are useful to reduce cardiovascular risk. Among the most commonly used lipid-lowering drugs are statins, fibrates, and in 1997 the FDA approved oats This research aimed to determine the effect of oats and lovastatin on lipid profile of dyslipidemic patients. The patient sample consisted of a total of 84 with dyslipidemia Tunjuelito hospital in Bogota. Were randomized and drug administration was conducted in randomly. Group 1 was administered Oats (A), 29 cases (34.5\%). Lovastatin+Avena Group $2(\mathrm{~A}+\mathrm{L}), 22$ cases $(26.2 \%)$ and Group 3 Lovastatin (L), 33 cases (39.3\%). They were followed for 12 weeks. The data were processed using SPSS. BMI showed no differences in group 1, being more significant in group 2 than in group 3. Waist circumference evidenced significant differences in the three groups. Cholesterol showed significant differences between group 1 and 3. Triglycerides showed significance in groups 2 and 3 . This study provides evidence that dyslipidemic patients benefit by adding a natural product like oatmeal on abdominal circumference parameters and cholesterol.

Key words: Lipid lowering, fibrates, abdominal circumference, colesterol.

\section{INTRODUCCIÓN}

Se ha postulado que las dietas ricas en fibras han sido útiles en disminuir el riesgo cardiovascular, independiente de la in- 
gesta calórica, contenido de grasa u otros factores dietarios (Davy et al. 2002). La prevalencia de hipertensión y obesidad en un estudio longitudinal de una Investigación Nutricional de Salud en Estados Unidos muestra que el Índice de Masa Corporal (IMC) ha aumentado en hombres y en mujeres, de 25,3 a 26,3kg/m², entre 1960 y 1991, con una ganancia promedio de $3,6 \mathrm{~kg}$. Paralelamente, se ha incrementado la ingesta de dietas para bajar de peso, comprendiendo vegetales, frutas, fibras y algunos fármacos, incluyendo, las estatinas (Saltzman et al. 2001a). La enfermedad cardiovascular (ECV) es la primera causa de morbimortalidad, tanto en hombres (43\%) como en mujeres (55\%), después de los 60 años, en países industrializados. En 1992, estas enfermedades produjeron el $40 \%$ de todas las defunciones, a nivel de Europa, ocupando, el primer lugar, las de origen cerebrovascular, con el $31,7 \%$, seguido de los infartos al miocardio, con el 26,6\% (Prieto et al. 1998). En nuestro medio, la tasa estimada por 100.000 habitantes, para 2004, fue para enfermedad isquémica cardiaca, de 76,6, en hombres y 67,1 en mujeres (Onatra et al. 2006). La etiología del daño es multifactorial, pero que, en general, es debida a formación de placa arterioesclerótica (Vaidya et al. 2011; Rullán Bonillas, 2002; Castaño Valencia, 2001).

Estatinas: Los medicamentos hipolipemiantes, se encuentran en la actualidad dentro del más usado, sobre todo las estatinas, seguido de los fibratos. Estos fármacos reducen los niveles de colesterol, lipoproteínas de baja densidad y pueden prevenir la formación de lesiones ateroscleróticas, lentificar su proceso y producir su regresión, mejorar la vasodilatación coronaria y reducir la mortalidad por cardiopatía coronaria. Las nuevas estrategias en la prevención de la enfermedad aterotrombótica, se centran en la evidencia aportada por el tratamiento hipolipemiante, tanto para prevención primaria como secundaria (Zhou \& Liao, 2010; Longyhore et al. 2009).

Avena: En 1999, el FDA aprobó, de manera relativa a la salud, afirmando que las dietas ricas en alimentos de granos integrales, también conocidos como granos enteros, podrían ayudar a reducir el riesgo de las enfermedades cardiovasculares. Recientes estudios indican que el consumir, por lo menos tres porciones por día de granos integrales, está asociado con un 20 a 30\% de disminución en el riesgo de enfermedades cardiovasculares y eventos cerebro vasculares (Swain et al. 1990). El grano o caropside está compuesto por dos estructuras principales, que son: el pericarpio, que contiene proteínas, lípidos y almidón (Shinnick et al. 1990) y la semilla, donde se encuentra la reserva de carbohidratos, en forma de almidón, cantidades mínimas de vitaminas, enzimas y ácidos grasos. La fibra es el principal contenido en la avena, que ayuda previniendo la absorción de lípidos, a nivel intestinal. La avena posee una de las fuentes más ricas de la fibra dietética soluble beta-glucano: de 5,0 (avena) a 7,2 (sal- vado de avena) gramos por cada ración de 100 g y ambas son fuentes importantes de fibra dietética total. Incluye más lípidos (5 - 9\%) que cualquier otro cereal y es rica en grasas poli-insaturadas, incluyendo, el écido graso esencial linoleico (Poppit, 2007).

Se han realizado estudios en humanos, en varios países, para evaluar los efectos que tiene el cereal frente a los lípidos, en donde se muestra una evidencia demostrando que ayuda a disminuir, en un 10-20\%, el colesterol total y LDL, en un 2-23\%. Estos resultados, se demostraron si la persona consume de 35 a $120 \mathrm{~g}$ de avena diario, en forma de salvado de avena, avena o goma de avena; a hombres y mujeres, el colesterol total disminuye un $10-20 \%$ y las LDL, en un $2-23 \%$ (Poppit, 2007). Existe evidencia que el efecto de la avena es mayor en pacientes hipercolesterolemicos que en pacientes normocolesterolemicos, mejorando el perfil lipídico, aumentando significativamente las concentraciones de HDL (Karl \& Saltzman, 2012).

Fue interés de los autores evaluar el efecto de la avena, lovastatina y avena+lovastatina sobre el perfil lipídico en pacientes que ingresaron a la consulta externa con dislipidemia, al hospital de Tunjuelito, en Bogotá D.C.

\section{MATERIAL Y MÉTODOS}

Se trata de un estudio de casos y controles aleatorizado, no enmascarado. La muestra incluyó 97 pacientes de ambos sexos, diagnosticados con dislipidemia, entre los 40 y 70 años de edad, quienes ingresaron a consulta externa de medicina interna, del Hospital de Tunjuelito II nivel, en el periodo del primero julio de 2008 al 31 julio del 2009 y que cumplieran con los criterios de inclusión y exclusión; 14 pacientes se retiraron voluntariamente. Criterios de Inclusión: 1) Paciente con dislipidemia; 2) Con edades entre 40 y 70 años de edad; 3) Que sean diagnosticados con dislipidemia por primera vez y, 4) Que no tomen la avena diariamente. Criterios de exclusión: 1) Menores de 40 años; 2) mayores de 70 años; 3) Pacientes con neoplasias; 4) Pacientes con enfermedad hepática crónica; 5) Pacientes con enfermedad renal y, 6) No autorización del consentimiento informado. Aspectos éticos: El estudio cumplió con la Resolución 8430/93 Ministerio de salud de Colombia (Normas científico-administrativas). Se presentó al Comité de Ética de la Universidad y al Comité de Investigaciones del Hospital. Riesgo mayor al mínimo (Experimental). Consentimiento informado: Fue firmado por todos los pacientes quienes ingresaron al estudio, con la posibilidad que pudieran renunciar cuando lo desee.

Las hipótesis alterna, postula variaciones de niveles de colesterol/ triglicéridos, en pacientes que padecen dislipidemia y que estén en tratamiento combinado (lovastatina+avena); hipótesis nula, que se mantienen igual las concentraciones 
de colesterol/triglicéridos, en pacientes que están con un sólo tratamiento (lovastatina o avena).

Para la recolección de la información, se tuvo en cuenta la elaboración del modelo de Historia clínica y perfil lipídico. En la identificación incluía: edad, estado civil, escolaridad, ocupación, nivel socio-económico, antecedentes familiares, antecedentes personales, como factores de riesgo cardiovascular, metabólico, estilo de vida, consumo de tabaco, consumo de medicamentos, frecuencia de ejercicio, dieta, examen físico y química sanguínea.

De los 84 pacientes para evaluar el efecto de la avena ( $3 \mathrm{~g} /$ día) y/o Lovastatina (20mg/día), durante doce semanas, se realizó la toma de perfil lipídico al inicio y al final del estudio. Se aleatorizaron tres grupos: Grupo 1 (Avena), 29 casos; Grupo 2 (Avena+Lovastatina), 22 casos y Grupo 3 (Lovastatina), 33 casos. Se formalizó historia clínica diseñada para el estudio, examen físico, el cual, incluyó peso, tensión arterial, perímetro abdominal, índice de masa corporal (IMC) (Peso/Talla2), toma de laboratorios, como perfil lipídico, que contenía niveles de colesterol total y triglicéridos. Se citaron los pacientes a tres consultas, cada una separada por cuatro semanas, para comparar anamnesis, examen físico y perfil lipídico. Luego, se procedió a tabular los resultados correspondientes a cada una de las variables del estudio, en el programa estadístico SPSS, donde se analizaron los resultados, determinando porcentajes, frecuencias representadas en tablas para cada variable y la prueba estadística ANOVA, para la evaluación intragrupos (Ruiz \& Morillo, 2004).

\section{RESULTADOS Y DISCUSIÓN}

En la Liga Colombiana contra la Enfermedad Coronaria y la Hipertensión Arterial, desde aproximadamente seis años, inició un estudio prospectivo, en las diferentes capitales de Colombia, incluyeron 53.245 pacientes, de los cuales, el 52\% eran hombres y el $47 \%$, mujeres; en cuanto a la distribución por raza, el $52 \%$ fue determinado como raza blanca y una franja de 48\%, como mestiza (Villarreal et al. 2008); un $8 \%$ de la población refiere antecedentes de enfermedad cerebrovascular y enfermedad coronaria; el 23\% de la población colombiana, según este estudio, presenta cifras de colesterol anormales, por encima de los $200 \mathrm{mg} / \mathrm{dl} \mathrm{y}$, el $12 \%$ cifras de triglicéridos, por encima de los $180 \mathrm{mg} / \mathrm{dl}$. La población, en un $47 \%$, no realiza ningún tipo de ejercicio; el $25 \%$ presentan sobrepeso y obesidad, postulándose un daño progresivo endotelial, acompañado de hipertensión y daño coronario (Villarreal et al. 2008).

En el presente estudio, el Grupo 1 (Avena sola), incluyó 29 casos, (34,5\%); Grupo 2 (Avena+Lovastatina), 22 casos (26,2\%) y, Grupo 3 (Lovastatina sola), 33 casos (39,3\%); la edad promedio fue de 53,51 años; el nivel socio-económico en este grupo es bajo, con predominio del estrato 2 (89,3\%); los antecedentes familiares evidencian que la enfermedad coronaria se presenta en el 65,5\%, hipertensión arterial $57,1 \%$, diabetes mellitus $39,3 \%$ y dislipidemia $66,6 \%$, cifras muy superiores al estudio de la Liga Colombiana contra la Enfermedad Coronaria e Hipertensión. En estilos de vida, fuman el 33,4\%, consume bebidas alcohólicas, el 67,8\% y no hace ejercicio, el $89,3 \%$, cifra muy superior a los datos del estudio sobre enfermedad coronaria, de $43 \%$. En relación con la dieta consumen en exceso harinas, $100 \%$ y fritos, 85,7\%; llama la atención que mantienen un consumo alto de verduras, carnes y lácteos (95\%). El uso de medicamentos en este grupo, como era de esperarse, maneja hipolipemiantes, $22,6 \%$, antihipertensivos, 15,5\% e, hipoglucemiantes, 9,5\%.

En relación a la variable peso y tratamiento, se realizó una prueba ANOVA, evidenciando que el grupo de Avena solamente aumentó $1,2 \%$ de peso, pero esta variación no es significativa $(\mathrm{p}<0.910)$; en el grupo de Avena $(\mathrm{A})+$ Lovastatina (L), se presenta un aumento leve de peso, de $1,1 \%$, sin ser significativo $(p<0.201)$. El grupo de Lovastatina únicamente disminuyó el peso en $1,8 \%$, con diferencia $(p<0.007)$ (Tabla 1).

En la evaluación del IMC, en el grupo que consumió (A sola), se presenta una diminución del $3 \%$, sin diferencia significativa entre el inicio y al final de tratamiento $(p<0.843)$; en el grupo de $(\mathrm{A}+\mathrm{L})$, la diminución fue del $4 \%$, con diferencia significativa al final del manejo $(\mathrm{p}<0.003)$ y el grupo de $(\mathrm{L}$ sola), evidencia una disminución del $5,1 \%$, con una significancia moderada $(\mathrm{p}<0.081)$ (Tabla 2). El promedio de sobrepeso (IMC > 31), se observó en el grupo 3 (L sola). En relación con el perímetro abdominal como medida indirecta del aumento de los lípidos, se reflejó una disminución del perímetro en los que consumieron (A), del 1\%, con diferencia significativa $(p<0.001)$; (L sola), una diminución del 2,1\%, con diferencia significativa $(\mathrm{p}<0.001) \mathrm{y}$, el grupo de $(\mathrm{A}+\mathrm{L})$, una disminución del $2 \%$, con una moderada diferencia significativa $(\mathrm{P}<0.016)$ (Tabla 3).

El aumento de colesterol, de las HDL, triglicéridos y disminución de la HDL tienden a aumentar en las mujeres después de la menopausia, atribuido, en parte, al efecto protector de los estrógenos en la modulación de la génesis de la arterioesclerosis. Este desnivel, se ve reflejado con el aumento de las patologías cardio o cerebro vasculares. En Estados Unidos cada año mujeres menores de 45 años empiezan ya a padecer de cardiopatías isquémicas; las mujeres sobrepasan a los hombres en eventos cardiovasculares, después de los 60 años; la mujer después de los 45 años tiene dos veces más de probabilidad de desarrollar diabetes mellitus, en comparación con el hombre. También, se puede hablar que las hiperlipidemias son principal causa de enfermedad y muerte en pacientes diabéticos (Berglund et al. 2012). 
Tabla 1. Variable de Peso inicial (I) y al final (F) del estudio.

\begin{tabular}{|c|c|c|c|c|c|c|}
\hline Grupo & $\mathbf{1}$ Toma (I) (DE) & $\mathbf{\%}$ & $\mathbf{2}$ Toma (F) (DE) & $\mathbf{\%}$ & Variación $\mathbf{~}$ & $\mathbf{P}<\mathbf{0 . 0 0 1}$ \\
\hline 1 (A) & $34,5+/-10,54$ & 34,5 & $34,9+/-10,35$ & 34,5 & +1.2 & 0,910 \\
\hline 2 (A+L) & $26,2+/-8,87$ & 26,2 & $26,5+/-9,03$ & 26,2 & +1.1 & 0,201 \\
\hline 3 (L) & $39,3+/-11,8$ & 39,3 & $38,6+/-11,76$ & 38,3 & -1.8 & $* * 0,007$ \\
\hline
\end{tabular}

*Desviación estándar

Tabla 2. Índice de Masa Corporal al inicio (I) y al final (F) del estudio.

\begin{tabular}{|c|c|c|c|c|c|c|}
\hline Grupo & $\begin{array}{c}\text { 1 Toma (I) } \\
\text { (DE)* }\end{array}$ & \% & $\begin{array}{c}\text { 2 Toma (F) } \\
\text { (DE)* }\end{array}$ & \% & $\begin{array}{c}\text { Variación } \\
\text { \% }\end{array}$ & $\mathbf{P}<\mathbf{0 . 0 0 1}$ \\
\hline $1(\mathrm{~A})$ & $27,9+/-3,96$ & 34,5 & $27,1+/-3,84$ & 34,5 & 3 & 0,843 \\
\hline 2 (A+L) & $27,3+/-2,97$ & 26,2 & $26,6+/-3,05$ & 26,2 & 4 & 0,003 \\
\hline $3(\mathrm{~L})$ & $31,04+/-4,37$ & 39,3 & $29,4+/-6,85$ & 39,3 & 5.1 & 0,081 \\
\hline
\end{tabular}

*Desviación estándar

Tabla 3. Perímetro abdominal inicial (I) y al final (F) del estudio.

\begin{tabular}{|c|c|c|c|c|c|c|}
\hline Grupo & $\begin{array}{c}\text { 1 Toma } \\
\text { (DE)* }\end{array}$ & \% & 2 Toma (DE) & \% & Variación \% & P< 0.001 \\
\hline $1(\mathrm{~A})$ & $97+/-11$ & 34,5 & $96,11+/-10$ & 34,5 & 1 & 0,001 \\
\hline $2(\mathrm{~A}+\mathrm{L})$ & $96+/-10$ & 26,2 & $94,10+/-9$ & 26,2 & 2 & 0,016 \\
\hline $3(\mathrm{~L})$ & $96+/-10$ & 39,3 & $94+10+/-9$ & 39,3 & 2.1 & 0,001 \\
\hline
\end{tabular}

*Desviación estándar

El Instituto Nacional de Salud (INS), entre 1977 y 1980, realizó la última medición de colesterol total en la población colombiana, donde se encontró que el 10,4\% de los hombres y el $16 \%$ de las mujeres presentaban elevados niveles de colesterol total, aproximadamente, en $240 \mathrm{mg} / \mathrm{dl}$. En la segunda encuesta realizada, se reflejan los factores de riesgo para enfermedades crónicas -ENFREC II-, donde se interrogó a la población estudiada sobre el antecedente de la toma de exámenes de colesterol y el tiempo (en años) trascurrido desde la última toma practicada y el resultado fue que uno de cada tres personas se tomó muestras de perfil lipídico (para la medición de HDL, LDL, VLDL y Triglicéridos), se habla de 1.353 personas, en un 76,9\% de la población estudiada (Rosano et al. 2007). Los valores de colesterol total se mantuvieron en unos rangos de $178 \mathrm{mg} / \mathrm{dl}$, en pacientes entre la edad de 18-39 años de edad; en personas de 40-69 años aumenta el rango, a un promedio de $192 \mathrm{mg} /$ dl. Esta elevación es mucho más notoria si se observa el grupo de las mujeres, por razones ya mencionadas anteriormente, cuyo promedio se basa en $171 \mathrm{mg} / \mathrm{dl}$, en mujeres de 18-39 años, aumentando el rango a $199 \mathrm{mg} / \mathrm{dl}$, en mujeres mayores de 40-69 años. Algunas mujeres estaban en los valores de colesterol, en $240 \mathrm{mg} / \mathrm{dl}$ (Rosano et al. 2007).

En el presente estudio, los niveles de colesterol total (CT), se encuentran por encima de los $200 \mathrm{mg} / \mathrm{dl}$ : al inicio y al final el grupo de Avena sola evidenció una disminución del 15\%, con diferencia significativa $(\mathrm{p}<0.003)$; Lovastaina sola, una diminución $6,4 \%(p<0.004)$. El grupo $(A+L)$ presentó una diminución del $17 \%$, sin diferencia significativa $(p<0.105)$ l (Tabla 4), datos concordantes con otro estudios (Longyhore et al. 2009).

En el estudio del INS, se muestra la prevalencia de hipercolesterolemia en la población colombiana, sea mujer u hombre, encontrándose que estas cifras se empiezan a elevar a medida que aumenta la edad. En estas condiciones, se deben mejorar los estilos de vida saludable, una dieta equi- 
Tabla 4. Toma de Colesterol Inicial (I) y al final (F).

\begin{tabular}{|c|c|c|c|c|c|c|}
\hline Grupo & $\begin{array}{c}\mathbf{1} \text { Toma (I) } \\
(\mathbf{D E})^{*}\end{array}$ & $\mathbf{\%}$ & $\begin{array}{c}\mathbf{2} \text { toma (F) } \\
(\mathrm{DE})^{*}\end{array}$ & $\%$ & Variación \% & $\mathbf{P}<\mathbf{0 . 0 0 1}$ \\
\hline $1(\mathrm{~A})$ & $213+/-50$ & 34,5 & $181+/-58$ & 34,5 & 15 & 0,003 \\
\hline $2(\mathrm{~A}+\mathrm{L})$ & $243+/-57$ & 26,2 & $201+/-65$ & 26,2 & 17 & 0,105 \\
\hline $3(\mathrm{~L})$ & $235+/-31$ & 39,3 & $220+/-29$ & 39,3 & 6.4 & 0,004 \\
\hline
\end{tabular}

*Desviación estándar

librada entre proteína, hidratos de carbono y verduras, una disminución en la ingesta de alcohol y tabaquismo, lo que colabora a que se presente mayor alteración a nivel del endotelio, favoreciendo a la placa ateromatosa, como ya se ha mencionado. El aumento de estos factores de riesgo favorecen la aparición de eventos cerebro vasculares y enfermedad coronaria (Rullán Bonillas, 2002).

Rosano et al. (2007) hallaron un promedio de colesterol de LDL de $112 \mathrm{mg} / \mathrm{dl}$, hablando de la población en total, en el grupo de 18-39 años fue de 108mg/dl y en el grupo de 40 69 años, aumentó a 121mg/dl. Esta diferencia de grupo es estadísticamente significativo $(p<0.001)$. Asimismo, como en el colesterol total se presenta un aumento evidente en el grupo de las mujeres, donde se observa un promedio elevado en comparación del grupo de los hombres, en las personas de 18-39 años, se manejan valores de $125 \mathrm{mg} / \mathrm{dl}$ y, en las mujeres, de 40-69 años, de $125 \mathrm{mg} / \mathrm{dl}$, lo que indica que, estadísticamente, también es significativo con $(\mathrm{p}<0.001)$ (Longyhore et al. 2009).

Teniendo en cuenta el aumento de la incidencia y la prevalencia de algún tipo de dislipidemia que indirectamente lleva a un daño progresivo endotelial, se podría realizar medidas de prevención, de detección y de tratamiento oportuno de los factores de riesgo, que pueden ser modificables. Evaluando los casos de dislipidemia, se puede promover, con mayor intensidad, el tratamiento no farmacológico, antes de iniciar un tratamiento, a través de programas poblacionales e individuales, haciéndole conocer, con mayor claridad, la información a las personas con factores de riesgo o personas que ya presenten alteraciones a nivel del perfil lipídico o clínicamente; sin embargo, es necesario aumentar el plan de conducta para lograr un diagnóstico precoz en la población colombiana, aun en sujetos al parecer sanos (Villarreal et al. 2008).

La dislipidemia es una de las entidades más frecuentes en la consulta regular en la población mayor de 40 años, en una alteración en la concentración de los lípidos, que puede ser de dos tipos: la hipercolesterolemia y la hipertrigliceridemia. La hipercolesterolemia es una alteración del transporte lipí- dico, que se produce por aumento en la síntesis o retraso en la degradación de las lipoproteínas, que transportan el colesterol y los triglicéridos del plasma (Kerchhoffs et al. 2003). Dado que la mayor parte del colesterol es transportado por las LDL, su alteración en la hipercolesterolemia se debe a un aumento de esta lipoproteína, donde la evidencia indica que las LDL modificadas, especialmente oxidadas, son atrapadas en la capa íntima de la arteria, siendo captadas por células, como los monocitos-macrófagos, a través de receptores que no tienen un sistema de autorregulación para el colesterol intracelular, transformándose en células espumosas, llenas de colesterol. Lo que conlleva a una inflamación de la pared arterial, asociada a disfunción del endotelio y este proceso de la placa de ateroesclerosis, lleva a la oclusión del lumen arterial. El efecto de la avena sola+lovastatina modifica, moderadamente, la diminución de lo triglicéridos, porque la lovastatina es conocido que lo disminuye.

Respecto a la hipertrigliceridemia, se atribuye a una mayor morbimortalidad coronaria, por su asociación con la disminución del colesterol HDL y por una modificación cualitativa de las LDL, que se transforman en partículas más pequeñas y más densas, que son más susceptibles a la oxidación y, por consiguiente, más aterogénicas (Ait-Oufella et al. 2011).

En la presenta investigación, se encuentra que los niveles de triglicéridos en el grupo de Avena sola presenta una variación del 27,4\%, sin diferencia significativa ( $<<0.176)$; en el grupo de $\mathrm{A}+\mathrm{L}$, la variación fue del $31,1 \%$, con diferencia levemente significativa $(p<0.051)$ y, en la que consumieron $\mathrm{L}$ sola, la variación fue del $5 \%$, con diferencia significativa $(\mathrm{p}<0.001)$ (Tabla 5), confirmando lo expresado en otras investigaciones, donde la Lovastatina disminuye los niveles de TG (Tobert, 2003).

Es claro que el manejo, de acuerdo a la clasificación de dislipidemia, las estatinas son los medicamentos de primera elección. Las estatinas, que son inhibidores competitivos de la 3-hidroxi-3-metilglutaril coenzima A (HMG-CoA) reductasa, enzima clave que regula la velocidad de biosíntesis del colesterol, aumentando el número de receptores de LDL y el catabolismo de estas lipoproteínas. Son las drogas más 
efectivas en reducir el nivel de Colesterol-LDL y, en altas dosis, disminuyen los triglicéridos. La principal acción de este grupo farmacológico es reducir los niveles de colesterol LDL, principal objetivo del tratamiento, en la mayoría de estos pacientes. Además de su efecto hipolipemiante, se han descrito otros efectos beneficiosos de las estatinas, tales como la es- tabilización de las placas de ateromas, capacidad antioxidante y mejoría de la función endotelial, previniendo el desarrollo de eventos cardiovasculares agudos. En este grupo, se encuentra la atorvastatina, la simvastatina y la lovastatina, que es la estatina más antigua y de menor potencia, para reducir los niveles de colesterol (Tobert, 2003).

Tabla 5. Toma de Trigliceridos Inicial (I) y al final (F).

\begin{tabular}{|c|c|c|c|c|c|c|}
\hline Grupo & $\begin{array}{c}\mathbf{1} \text { Toma (I) } \\
\text { (DE)* }\end{array}$ & $\%$ & $\begin{array}{c}\mathbf{2} \text { toma (F) } \\
\text { (DE)* }\end{array}$ & $\%$ & Variación \% & $\mathbf{P}<\mathbf{0 . 0 0 1}$ \\
\hline $1(\mathrm{~A})$ & $260+/-113$ & 34,5 & $188+/-89$ & 34,5 & 27.4 & 0,176 \\
\hline $2(\mathrm{~A}+\mathrm{L})$ & $273+/-106$ & 26,2 & $185+/-83$ & 26,2 & 31.1 & 0,051 \\
\hline $3(\mathrm{~L})$ & $264+/-138$ & 39,3 & $251+/-138$ & 39,3 & 5 & 0,001 \\
\hline
\end{tabular}

*Desviación estándar

La dieta-terapia con productos ricos en fibra soluble e insoluble, como la avena, ha disminuido las concentraciones plasmáticas del colesterol total y las LDL; estas dietas están diseñadas para reducir, en forma progresiva, la ingesta de ácidos grasos saturados, colesterol y también promover una baja de peso en aquellos pacientes con sobrepeso, a través de la eliminación de un exceso de calorías totales. Para alcanzar una ingesta diaria de fibra, dentro de los rangos recomendados, se debe consumir 5-6 porciones de frutas o verduras más alimentos del grupo de los cereales, papas y leguminosas, ricos en fibra. En la hipertrigliceridemia, las medidas no farmacológicas son similares a las descritas para la hipercolesterolemia; sin embargo, el énfasis debe estar en la reducción de peso, la disminución del consumo de azúcares refinados y fructosa, la disminución del consumo de alcohol y suprimir el tabaquismo. En relación con la dieta de avena en hojuelas existen dos tipos: la roja y la blanca. Estas dos clases pueden disminuir las LDL plasmáticas, ya que poseen un excelente componente de fibra soluble e insoluble y no permiten la absorción del colesterol a nivel intestinal, pero sí su eliminación, a través de las heces (Behall et al. 2004).

Es conocido que la hipertensión, la dislipidemia y el sobrepeso son entidades que aumentan el riesgo cardiovascular (RCV). En el presente estudio, se destaca la alta prevalencia dentro de los antecedentes de: dislipidemia, 66,6\%; enfermedad coronaria, 65,5\%; hipertensión, 57,1\% y diabetes, $39,3 \%$. Para mejorar el riesgo, se han propuesto diferentes medidas farmacológicas-anhipertensivos, hipolipemiantes, vasodilatadores $\mathrm{y} / \mathrm{o}$ beta/bloqueadores, diuréticos y no farmacológicas, como el ejercicio, la dieta hipocalórica, demostrándose como una dieta rica en fibra y avena, que disminuye la presión arterial, mejora el perfil lipídico y disminuye el peso (Saltzman et al. 2001b). Los datos demuestra que existe una disminución de peso de 1 a $2 \mathrm{~kg}$, con dife- rencia significativa $p<0.007$, especialmente, con la asociación $\mathrm{L}+\mathrm{A}$. La evaluación del perímetro abdominal presenta un diminución entre 1 y $2 \%$, evidenciando una disminución significativa $p<0.001$, especialmente, con la A sola y la $\mathrm{L}$ sola, estudios a largo plazo muestran disminución (Saltzman et al. 2001b).

En un estudio parecido (Jones et al. 2005), compararon el efecto de las Lovastatina $(20 \mathrm{mg})$ y un portafolio de dieta a base de esteroles $(1 \mathrm{~g} / 1000 \mathrm{Lcal})$, proteínas de soya $(21,4 \mathrm{~g} / 1000 \mathrm{Lcal})$, almidones $(14 \mathrm{~g} / 1000 \mathrm{Kcal})$, fibra, avena, vegetales $(10 \mathrm{~g} / 1000 \mathrm{~kg})$, sobre los niveles de colesterol. Manejaron una muestra de 34 pacientes, 20 hombres y 14 mujeres, con criterios de inclusión, como: elevación del colesterol y triglicéridos a nivel sanguíneo, edad en hombres $>55$ y mujeres $>62$ años de edad, con perímetro abdominal $>77 \mathrm{~cm}$ y un peso en hombres de $82 \mathrm{~kg}$ en promedio y en mujeres de 67. Se tuvo en cuenta, como criterios de exclusión, que no presentaran ningún tipo de enfermedad a nivel renal, cardiovascular, diabetes e hipertensión. Además, no estuviesen en el momento en ningún tipo de tratamiento, que disminuyera los niveles, tanto de colesterol como de triglicéridos y que las mujeres no se encontraran en manejo de reemplazo hormonal. Posterior a esto, los pacientes con los criterios de inclusión fueron sometidos a un examen físico, donde se tuvo en cuenta las siguientes variables: peso, perímetro abdominal y tensión arterial de manera rutinaria en sus tres periodos, exámenes de laboratorio, midiendo colesterol total, HDL, LDL y triglicéridos, de manera secuencial. Se evidenció que el colesterol LDL disminuyó 8,5\% (Estatinas) y $33 \%$, con fibras y vegetales $(p<0.013)$, sin cambios significativos, entre las estativas y la fibra/avena/vegetales. En el presente estudio, la avena disminuyó el colesterol total $15 \%$ y la lovastatina sola, el $6,4 \%$, con resultado parecidos a los de Jones et al. (2005). 
De igual manera, en este trabajo, se manejaron criterios de inclusión parecidos, en cuanto a la edad y la evaluación de los niveles de colesterol y triglicéridos a nivel sanguíneo. Como criterios de exclusión, en este estudio, se quería pacientes que no tuvieran antecedentes de enfermedad hepática y renal y no estuviesen actualmente tomando ningún tipo de tratamiento hipolipemiante. Al hacer el estudio comparativo, se demuestra cómo existe una disminución en los niveles de colesterol más evidentes en el régimen $(A+L)$ y (A) $\mathrm{p}<0.013$. Los triglicéridos, si bien no hubo diferencias significativas en el primer control, al inicial de las cuatro semanas, se evidencia una disminución significativa $p<0.32$, especialmente, con el régimen $(L+A)$. En conclusión, se demuestra una eficacia mayor en el tratamiento conjunto de dieta rica en fibra, de manera coadyuvante con las estatinas, que con las estatinas sola, como lo mencionan Jula et al. (2002), Katz et al. (2004) y Maki et al. (2007).

En comparación con otro estudio realizado por Jenkins et al. (2005), en el 2000, quienes evaluaron los efectos de la avena (60g/día), antioxidantes (Vitamina E 400 mg + Vitamina C $500 \mathrm{mg}$ ) vs. placebo y la función endotelial, midiendo la vasodilatación mediada por flujo FMD), con escáner la reactividad de la arteria braquial (BARS), en adultos con sobrepeso y dislipidemia. Incluyeron pacientes entre los 35-70 años de edad, 16 hombres >35 anos, 14 mujeres postmenopáusicas sin uso de tratamiento de remplazo hormonal, no fumadores, pacientes sin antecedente conocido de enfermedad coronaria o vascular, sin manejo vaso activo y con colesterol $>200$ y triglicéridos $>150$, en forma aleatorizada y durante seis semanas. Los autores sugieren, pero no confirman, el escaso beneficio sobre la dislipidemia, sobrepeso y función endotelial de la avena, sugiriendo un aumento de la muestra para futuros estudios.

En conclusión, se puede afirmar que el presente estudio demuestra cómo pacientes dislipidémicos se benefician al agregar un producto natural, como la avena, a su programa terapéutico de lovastatina. Se sugiere aumentar el número de casos y su seguimiento a largo plazo. A pesar de un tiempo tan corto, se evidencia una disminución en los valores de perímetro abdominal en los tres grupos y un efecto moderadamente significativo sobre los niveles de colesterol y triglicéridos, en asocio con lovastatina, que cuando se consume avena sola.

Agradecimientos: Los autores agradecen a las directivas de la Universidad y de la Facultad de Medicina, por el apoyo recibido en el desarrollo de esta investigación. Conflicto de intereses: El manuscrito fue preparado y revisado con la participación de todos los autores, quienes declaramos que no existe ningún conflicto de intereses, que ponga en riesgo la validez de los resultados presentados.

\section{BIBLIOGRAFÍA}

1. AIT-OUFELLA, H.; TALEB, S.; MALLAT, Z.; TEDGUI, A. 2011. Recent advances on the role of cytokines in atherosclerosis. Arterioscl. Thromb. Vas. Biol. 31(5):969-979.

2. BEHALL, K.M.; SCHOLFIELD, D.J.; HALLFRISCH, J. 2004. Diets containing barley significantly reduce lipids in mildly hypercholesterolemic men and women. Am. J. Clin. Nutr. 80(5):1185-1193.

3. BERGLUND, L.; BRUNZELL, J.D.; GOLDBERG, A.C.; GOLDBERG, I.J.; SACKS, F.; MURAD, M.H.; STALENHOEF, A.F. 2012. Evaluation and treatment of hypertriglyceridemia: an endocrine society clinical practice guideline. J. Clin. Endocrinol. Metab. 97(9):2969-2989.

4. CASTAÑO VALENCIA, O. 2001. Factores de riesgo en la ciudad de Manizales. Archivos de Medicina. Universidad de Manizales. 1(3):17-22.

5. DAVY, B.M.; DAVY, K.P.; HO, R.C.; BESKE, S.D.; DAVRATH, L.R.; MELBY, C.L. 2002. High-fiber oat cereal compared with wheat cereal consumption favorably alters LDL-cholesterol subclass and particle numbers in middle-aged and older men. Am. J. Clin. Nutr. 76(2):351-358.

6. JENKINS, D.J.A.; KENDALL, C.W.C.; MARCHIE, A.; FAULKNER, D.A.; WONG, J.M.W.; DE SOUZA, R.; EMAM, A.; PARKER, T.L.; VIDGEN, E.; TRAUTWEIN, E.A.; LAPSLEY, K.G.; JOSSE, R.G.; LEITER, L.A.; SINGER, W.; CONNELLY, P.W. 2005. Direct comparison of a dietary portfolio of cholesterol-lowering foods with a statin in hypercholesterolemic participants. Am. J. Clin. Nutr. 81(2):380-387.

7. JONES, P.J.; RAEINI-SARJAZ, M.; JENKINS, D.J.; KENDALL, C.W.; VIDGEN, E.; TRAUTWEIN, E.A.; LAPSLEY, K.G.; MARCHIE, A.; CUNNANE, S.C.; CONNELLY, P.W. 2005. Effects of a diet high in plant sterols, vegetable proteins, and viscous fibers (dietary portfolio) on circulating sterol levels and red cell fragility in hypercholesterolemic subjects. Lipids. 40(2):169-174.

8. JULA, A.; MARNIEMI, J.A.; HUUPPONEN, R.; VIRTANEN, A.; RASTAS, M.; RÖNNEMAA, T. 2002. Effects of diet and simvastatin on serum lipids, insulin and antioxidants in hypercholesterolemic men:a randomized controlled trial. JAMA. 287(5):598-605. 
9. KARL, J.P.; SALTZMAN, E. 2012. The role of whole grains in body weight regulation. Adv. Nutr. 3(5):697-707.

10. KATZ, D.L.; EVANS, M.A.; CHAN, W.; NAWAZ, H.; COMERFORD, B.P.; HOXLEY, M.L.; NJIKE, V.Y.; SARREL, P.M. 2004. Oats, antioxidants and endothelial function in overweight, dyslipidemic adults. J. Am. Coll. Nutr. 23(5):397-403.

11. KERCHHOFFS, D.; HORNSTRA, G.; MENSINK, R.P. 2003. Cholesterol-lowering effect of glucan from oat bran in mildly hypercholesterolemic subjects may decrease when glucan is incorporated into bread and cookies. Am. J. Clin. Nutr. 78:221-227.

12. LONGYHORE, D.S.; STOCKTON, C.M.; THOMAS, M.R. 2009. Profile changes associated with changing available formulary statins: removing higher potency agent. Am. J. Manag. Care. 15(7):409-414.

13. MAKI, K.C.; DAVIDSON, M.H.; WITCHGER, M.S.; DICKLIN, M.R.; SUBBAIAH, P.V. 2007. Effects of highfiber oat and wheat cereals on postprandial glucose and lipid responses in healthy men. Int. J. Vitam Nutr. Res. 77(5):347-356.

14. ONATRA, W.; GARCÍA, C.; GAMBOA, C.; SÁNCHEZ, J.; CASTRO, J. 2006. Prevalencia del síndrome metabólico en mujeres posmenopausicas en dos centros clínicos de Bogotá. Rev. U.D.C.A Act. \& Div. Cient. 9:43-53.

15. POPPIT, S.D. 2007. Soluble fibre oat and barley $\beta$-glucan enriched products: can we predict cholesterol-lowering effects? Br. J. Nutr. 97(6):1049-1050.

16. PRIETO ALBINO, L.; ARROYO DÍEZ, J.; VADILLO MACHOTA, J.M.; MATEOS MONETERO, C.; GALAN REBOLLO, A. 1998. Prevalencia de hiperlipidemia en niños y adolescentes de la provincia de Cáceres. Rev. Española Salud Publ. 72:343-355.

17. ROSANO, M.; VITALE, C.; TULLI, A. 2007. Manejo del riesgo cardiovascular en mujeres postmenopausicas. Rev. Col. Menopausia 13(1):25-28.

18. RUIZ, R.M.; MORILLO, L.E. 2004. Epidemiologia Clínica: Investigación clínica aplicada. Ed. Panam. BogotaMadrid-Sao Paulo. p.181-194.
19. RULLÁN BONILLAS, A. 2002. La avena y sus efectos hipocolesterolemicos. Nutr. Clin. 5(3):139-146.

20. SALTZMAN, E.; MORIGUTI, J.C.; DAS, S.K.; CORRALES, A.; FUSS, P.; GREENBERG, A.S.; ROBERTS, S.B. 2001a. Effects of a cereal rich in soluble fiber on body composition and dietary compliance during consumption of a hypocaloric diet. J. Am. Coll. Nutr. $20: 50-57$

21. SALTZMAN, E.; DAS, S.K.; LICHTENSTEIN, A.H.; DALLAL, G.E.; CORRALES, A.; SCHAEFER, E.J.; GREENBERG, A.S.; ROBERTS, S.B. 2001b. An oat-containing hypocaloric diet reduces systolic blood pressure and improves lipid profile beyond effects of weight loss in men and women. J. Nutr. 131(5):1465-1470.

22. SHINNICK, F.L.; INK, S.L.; MARLETT, J.A. 1990. Dose response to a dietary oat bran fraction in cholesterol fed rats. J. Nutri. 120(6):561-568.

23. SWAIN, J.F.; ROUSE, I.L.; CURLEY, C.B.; SACKS, F.M. 1990. Comparison of the effects of oat bran and lowfiber wheat on serum lipoprotein levels and blood pressure. New Engl. J. Med. 322(3):147-152.

24. TOBERT, J.A. 2003. Lovastatin and beyond: the history of the HMG-CoA reductase inhibitors. Nat. Rev. Drug Discov. 2(7):517-526.

25. VAIDYA, D.; SZKLO, M.; CUSHMAN, M.; HOLVOET, P.; POLAK, J.; BAHRAMI, H.; JENNY, N.S.; OUYANG, P. 2011. Association of endothelial and oxidative stress with metabolic syndrome and subclinical atherosclerosis: multi-ethnic study of atherosclerosis. Eur. J. Clin. Nutr. 65(7):818-825.

26. VILLARREAL, E.; FORERO, Y.; POVEDA, E.; BARACALDO, C.; LÓPEZ E. 2008. Marcadores de riesgo cardiovascular en escolares de cinco departamentos de la región oriental en Colombia. Biomedica. 28(1):38-49.

27. ZHOU, Q.; LIAO, J.K. 2010. Pleiotropic effects of statins: Basic research and clinical perspectives. Circ. J. 74(5):818-826.

Recibido: Julio 3 de 2013

Aceptado: Octubre 9 de 2013

Como citar:

Alarcón Galeano, A.M.; Buitrago Hernández, D.F.; Romero Fierro, L.A.; Sánchéz Leyva, A.M.; Onatra H., W.; Rios, M.T. 2013. Efecto de la avena y/o lovastatina sobre el perfil lipídico en pacientes dislipidémicos del Hospital de Tunjuelito, Bogotá 20082009. Rev. U.D.C.A Act. \& Div. Cient. 16(2): 319-326. 